\title{
Deviation Management System - A Boon in Industrial Quality Sciences for Compliance
}

\author{
Seetha Ram Kotra ${ }^{1 \#}$, Rajeswari Ramayanam ${ }^{2}$, Lavanya Reddy ${ }^{3 \#}$, Sudheer Reddy \\ Billipelli $^{4 \#}$, Ganesh Tsavatapalli ${ }^{1 \#}$, Daya Thota ${ }^{5}$, Sandhya Prathapaneni ${ }^{6 \#}$ and PVD \\ Sowjanya Kumari ${ }^{7^{*}}$ \\ ${ }^{1}$ Department of Biotechnology, Acharya Nagarjuna University, Guntur - 522510 \\ ${ }^{2}$ Department of Physics, Acharya Nagarjuna University, Guntur - 522510 \\ ${ }^{3}$ Department of Biotechnology, The Oxford College of Engineering, \\ Bangalore - 560068 \\ ${ }^{4}$ Department of Microbiology, SVS PG College, Kakatiya University, \\ Warangal - 506009 \\ ${ }^{5}$ Department of Microbiology, Sri Venkateswara University, Tirupati - 517502 \\ ${ }^{6}$ Department of Chemistry, Sri Padmavati Mahila Visvavidyalayam, \\ Tirupati - 517502 \\ ${ }^{7}$ Department of Microbiology, MVR \& PG College, Vishakapatnam - 530026 \\ *pvdsk_16@yahoo.com
}

\begin{abstract}
Production of quality products in biopharmaceutical companies can take up a principle of scientific, logical, contamination free, risk and impact assessment based approaches. All product deliverable industries come under Good Manufacturing Practices (GMP) which will be duly audited by different regulatory agencies across the world. Identification of instruction which departs from the approved written procedure is called deviation. End user from user department has to identify the deviation during manufacturing process and report to QA (Quality Assurance) for further action. After commenting on deviation by user department head, QA should mark the relevant departments for their comments on deviation and impact assessment. Based on the investigation, impact to be assessed and deviation will be classified by QA as minor/ major/ critical. Initial classification of the deviation need not be in inline with final classification. Appropriate corrective action and preventive action (CAPA) will be proposed by user department with the co-ordination of relevant cross functional teams that will be duly verified and approved by QA. CAPA implementation will be carried out if applicable. Once after initiating the CAPA, deviation will be closed and CAPA effectiveness will be tracked. This is the first report on industrial deviation practices for compliance.
\end{abstract}

Keywords: Deviation, cross functional teams, investigation, root cause, corrective action preventive action and close out

\section{Introduction}

All sterile manufacturers should meet the requirements of current good manufacturing practices of different regulatory agencies. GMP (Good Manufacturing Practices) facilitated biopharmaceutical companies are under the category of scientific and risk-

Received (January 17, 2018), Review Result (May 1, 2018), Accepted (May 11, 2018)

\# Authors have equal contribution

* Corresponding Author 
based approaches. This document gives description of procedure for dealing of non conformance activities during all stages from manufacturing to market complaints. Concept of the deviation is to document the non conformance and to ensure that all non conformances are reported and investigated thoroughly to assess the impact on product quality [1]. Deviation procedure is applicable for developmental/ engineering/ clinical/ pre clinical/ process validation/ commercial batches in best industrial practices [2]. Any non conformance/ deviating the approved instructions of written procedures are called deviation.

User department is responsible for the identification, reporting and investigation of the non conformance. Quality assurance is responsible for the deviation management at all stages; in view of deviation log in, investigation, review and co-ordination in CAPA proposal and close out as well. Other cross functional teams will review the deviation investigation or will involve in the investigation where and when required. National and international regulatory agencies will review the impact on regulatory requirements of different markets and on continuous quality improvements [3]. Deviation will be initiated for any type of wrong entry or entry error or missed entry where supporting documents are not available. Failing to submission of supporting documents along with batch manufacturing record or checklist, deviation will be triggered by user department.

\section{A Proactive Approach to Continuous Quality Improvement (CQI)}

Deviation management is a continuous quality improvement process consisting of planning, where and when an opportunity for improvement is required. Based on the planning, data collection will be acquired. Further, operator has to analyze the deviation with appropriate investigational tool and perform impact assessment.

\section{Flowchart of Deviation Path}

Deviation flow chart depicting the steps to be followed from initiation to close out is described in the following Figure 1.

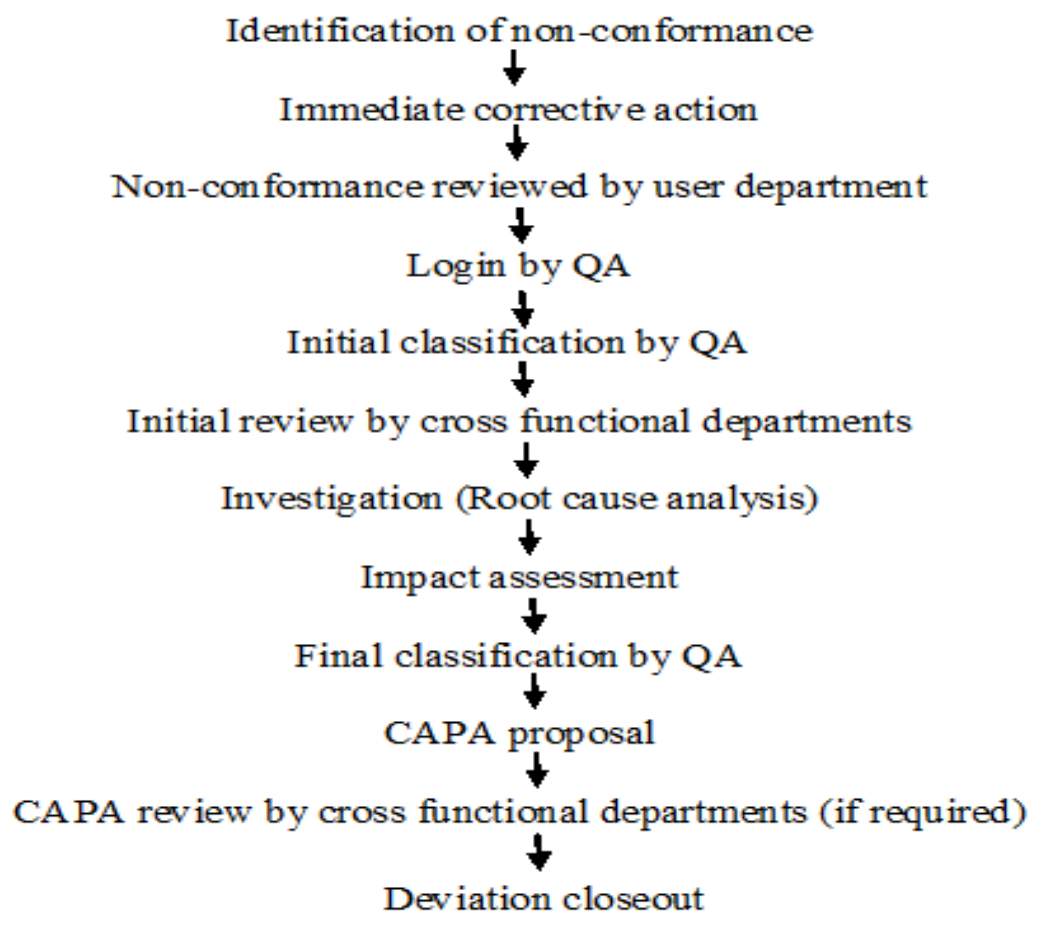

Figure 1. Deviation Management Flow Chart 


\section{Types of Deviation}

Deviations are of two types based on the event or event to be carried out i.e., planned and unplanned deviations. In an effective quality management system, all planned and unplanned deviations will be closed within 30 working days with a window period of 10 working days. For every unplanned critical/ major deviations impact assessment will be carried out post to activity.

\subsection{Planned Deviation}

Departure from approved written instruction, before start of the activity for continuous quality improvement purpose is called planned deviation. All planned deviations will not alter the quality of product, so all planned deviations are classified as minor. For all planned deviations proper evaluation and risk assessment is required. All planned deviations should be duly justifiable in view of product integrity/ product quality. Planned deviations are duly verified by national and international regulatory agencies without fail (even minor) and should be closed before batch release. Planned deviations of similar nature should not be repeated for more than three batches for same or different products of same or different stage. For every planned deviation, impact assessment to be carried out before initiation of deviation. If recurring deviations are observed, systems are not stringent and appropriate preventive actions will be taken by higher authorities in view of compliance. At every stage of planned deviations, user department personnel should provide the compliance data up to final step of the process.

\subsection{Unplanned Deviation}

Violation of written procedures during the batch or observation of violation of written procedure post execution of activity is called unplanned deviation. All unplanned deviations are properly reported to QA and all unplanned deviations are investigated for proper root cause with different investigation tools where and when required. These unplanned deviations may be critical/ major/ minor. For all unplanned deviations corrective action and preventive action is mandatory to nullify the recurrence of noncompliance in view of good manufacturing practices.

\section{Classification}

\subsection{Critical Deviation}

Deviation likely to have significant impact on product quality is called critical deviation. The following are the examples of critical deviations, but not limited to

i. Operator does not follow critical process parameters during manufacturing.

ii. Usage of unapproved/ expired/ rejected materials in manufacturing.

iii. Printing the wrong batch numbers on labels.

iv. Identifying the data integrity issues.

v. Usage of raw materials in lesser quantities in manufacturing operations/testing's.

vi. An observation resulting in a significant risk when used in a finished product.

vii. Batch contamination/ sterility breach

\subsection{Major Deviation}

Deviations which may have impact on critical attributes of the product quality is called major deviation. The following are the examples of critical deviations, but not limited to 
i. Receiving of raw materials in damaged containers

ii. Line clearance was not ensured for product change over

iii. Involving in manufacturing operations without training.

iv. Using non qualified equipments for analysis.

v. Violation in aseptic behaviour

vi. Out of limits of relative humidity, temperature and differential pressure in clean rooms

vii. Performing the preventive maintenance beyond the schedule.

viii.Process yield is not within the range.

ix. Use of equipment which is under OOC (Out of calibration)

x. Critical process parameters (CPP) was not monitored

xi. Use of unapproved reference standard.

xii. Operators fails to ensure the clean room behaviour during manufacturing

xiii. Analysis of stability samples beyond schedule time

xiv. Skipping of critical steps mentioned in SOP (Standard Operating Procedure)/ ECC (Equipment Cleaning Checklist)/GAM (General Analytical Method)

\subsection{Minor Deviation}

Deviations which do not have any impact in any form on the product quality is called minor deviation. The following are the examples of critical deviations, but not limited to

i. Line clearance not taken for batch change over

ii. Not following FIFO (First In First Out)/ FEFO (First Expiry First Out)

iii. Wrong/missed/entry errors which not affect the product quality

iv. Out of pressure differentials in non critical areas

v. Handling the materials/solutions without proper labelling

vi. Proposed changes are not implemented

\section{Deviation Reporting}

All deviation forms should be duly issued by QA upon request by user department head/ in-charge/ designee and to be documented for review and trending of management review meeting respectively (Annexure - 1). Further, user department personnel will write the deviation details including the date of occurrence and along with date of identification (Annexure - 2). Those details will be reviewed by user department head and should confirm the noncompliance against standard.

Any non-compliance shall be reported at earliest to QA without any delay. Industries which are under cGMP (current Good Manufacturing Practices) will report the non compliance within 24 hours. Deviations will be initiated by initiator or who identifies the non compliance irrespective of department. During initiation, what is standard and what is non compliance should be stated clearly along with the reference document number, if applicable. After observation of non compliance, what immediate corrective action is taken by user department personnel/ initiator to nullify the impact on product quality should be stated accurately. If immediate corrective action does not nullify the noncompliance, then what additional immediate corrective action is taken against to deviation 
will be provided along with proper justification. Each and every non compliance, should be routed through separate deviation including same type of non compliance. But investigations of similar deviations have to merged in a single investigation. If a document is erroneous, before initiating, user department personnel/ QA can replace/ demand for the replacement of document by attending the comments. For replacement of any type of document which is erroneous and was not enrolled in QA records, deviation is not necessary. Effective quality system should build based on ALCOA (Attributable, Legible, Contemporaneous, Original and Accurate) or ALCOA+ (Attributable, Legible, Contemporaneous, Original and Accurate, Complete, Consistent, Enduring and Available).

\section{Deviation Log In and Initial Classification}

For every deviation, QA SME (Subject Matter Expert) has to verify the details and what are the immediate actions taken for that non compliance and should be logged in the register (Annexure - 3). Later, QA has to review the similar deviations and impact of current deviation on product quality in any manner should be verified and deviation will be classified as critical or major or minor. But initial classification is not the final one. In case of critical deviations, if user department want to take next batch immediately, an interim report will be prepared, reviewed by cross functional teams and should be approved by QA before next batch start up.

\section{Cross Functional Teams Review}

Deviation details reviewed by QA will be shared to cross functional teams by the initiator as per relevance. Concern cross functional departments should review the deviation details and has to provide their comments on non-conformance. For impact on product quality, comments from manufacturing sciences and R\&D (Research \& Development) are required. For impact on product quality on regulatory markets, NRS (National Regulatory Sciences) and IRS (International Regulatory Sciences) comments are required. Based on the cross functional team comments, QA will demand the investigation with risk assessment if any required.

\section{Investigation by User Department}

A process investigation is a guideline to ensure that it was triggered by user department for product or process or equipment deviation which follows a logical investigation process using suitable investigation techniques to sort out root cause. These investigation tools can be used not only for deviations, but also for batch contaminations, audit observations, out of specifications, out of trends and, product complaints and recalls. Selection of specific root cause analysis tools is purely case specific. QA will co-ordinate for any type of investigation. For efficient quality management system, investigation of a deviation and subsequent determination of root cause are the fundamental requirements. To avoid the recurrences, a thorough investigation is preferred, where an incomplete investigation provides no proper root cause and may cause reoccurrence. It is very important to perform an impact assessment of non compliance when it occurs. Effective deviation management handling does not require amendment. If any gaps identified in investigation of closed deviation, a separate deviation will be triggered to overcome the gap in view of compliance.

Ideal investigation conducted at the time of occurrence of non compliance but not limited to observation of non conformance after the event occurs. Employee is at place or operator identifies the indication of non compliance, investigation to be carried out using interview process or collecting the evidences or pictograms or FMEA (Failure Mode and Effect Analysis) or 5 Whys technique or meeting technique or fish bone analysis. For non 
conformance is noted in analytical tests or in stability testing or anomaly noted during periodic review of document, investigation to be carried out using $5 \mathrm{~W} \& 1 \mathrm{H}$ or 5 whys techniques or fish bone analysis. Investigation tools may be of different types.

\subsection{Process Open Flow Chart}

A visual representation of process open flow chart techniques is useful as a starting point for most of the investigations (Figure 2). In most of the deviations, it facilitates the identification of root cause.

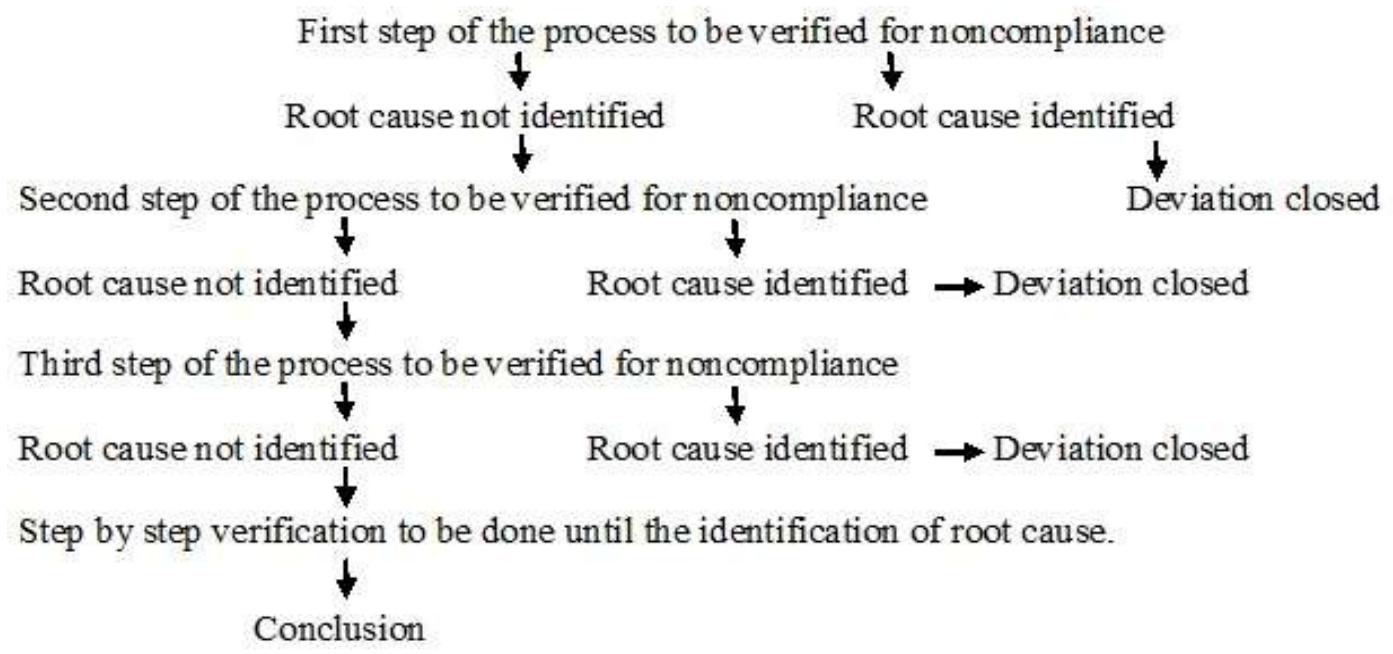

Figure 2. Detailed Process Open Flow Chart

\subsection{Fish Bone Analysis}

A fishbone diagram is a visualization tool for categorizing the probable or potential causes of a deviation in order to recognize the root cause. To identify the root cause for all unknown deviations, Japanese quality control expert, Ishikawa proposed a model, which is familiar with the name of fish bone analysis (Figure 3). This the method to identify and explore of all possible causes of a deviation. In this method, equipment, measurement, people, environment, methods and material can be analysed for the root cause. If the process related deviation occurs, process stages will become the part of fishbone analysis.

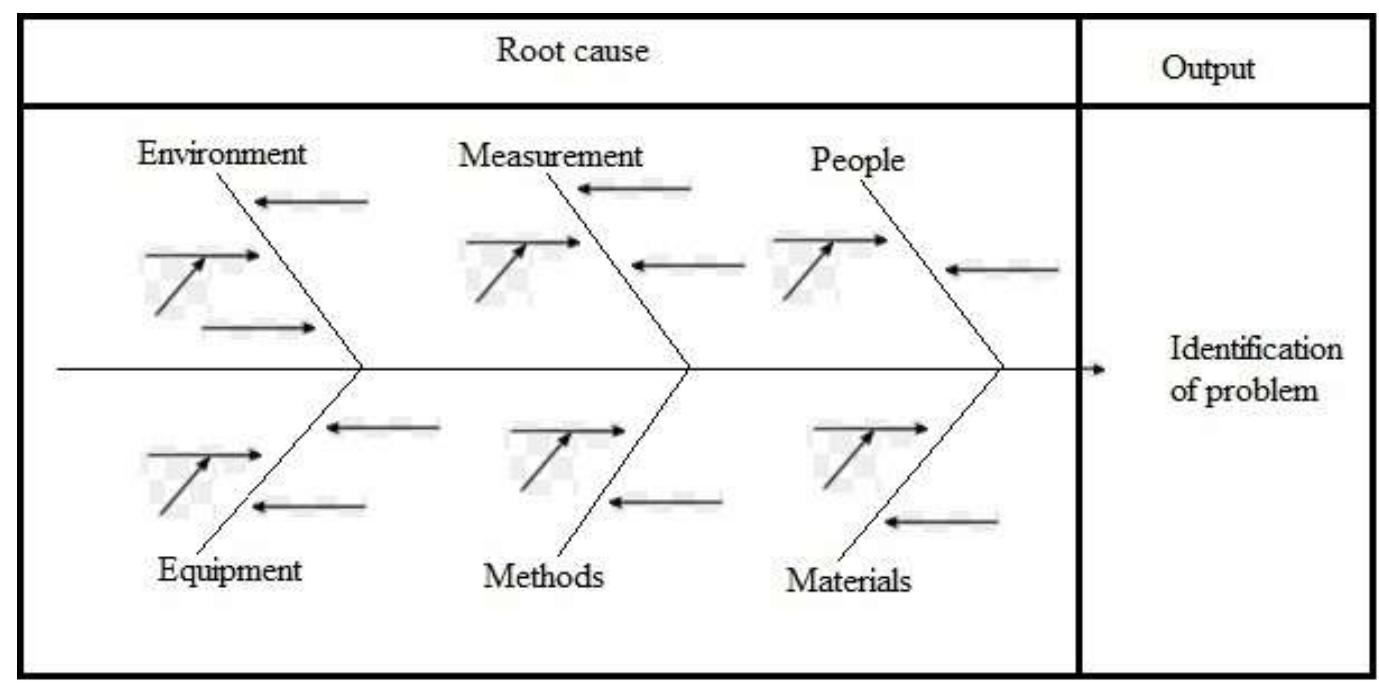

Figure 3. Ishikawa Fish Bone Analysis Tool 


\subsection{Five Why's}

To identify the root cause of a deviation, five why's technique shall be used in connection with the cause and effect diagram to probe more deeply (Figure 4). This method of analysis will probably provide the root cause for the non compliance. This method is used to conduct the investigation in the following procedures.

i. Determine the starting point where the non compliance to be analysed further.

ii. Logical thinking to sort out the root cause before the starting of activity

iii. For every identified cause, question the system like "why this is the cause for non compliance"

iv. System should be questioned repeatedly until the identification of root cause.

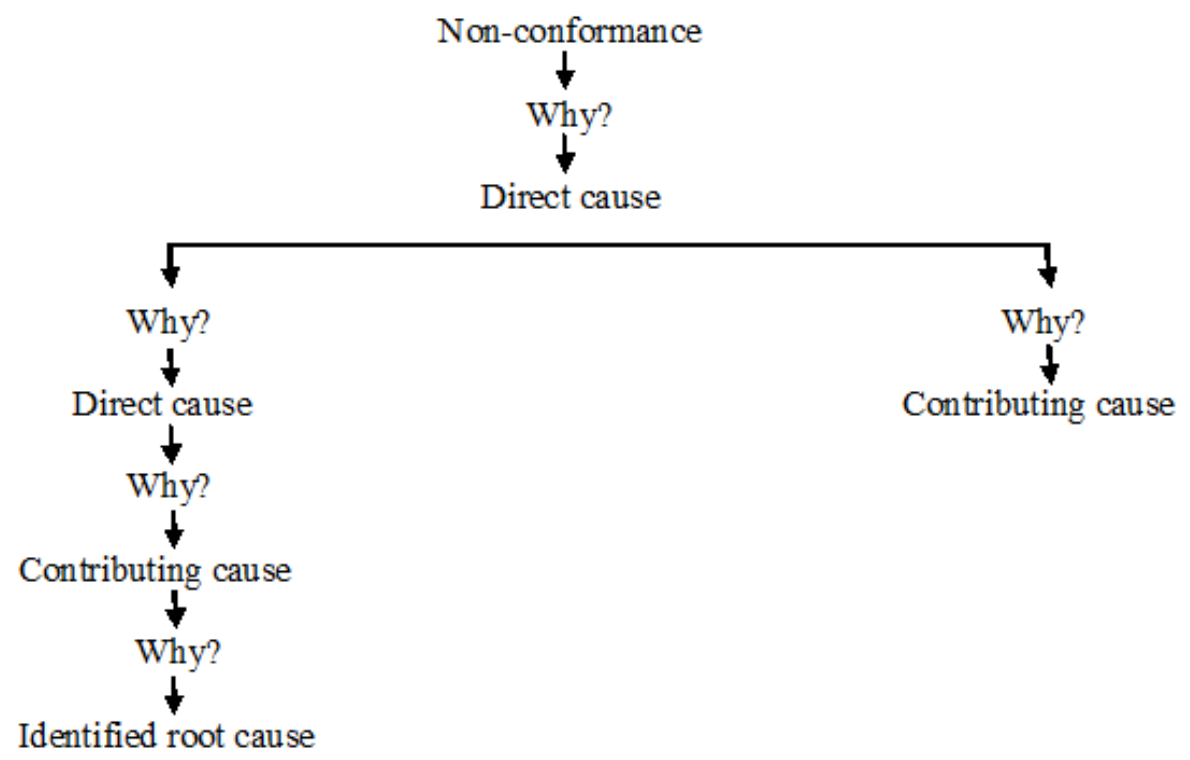

Figure 4. 5 Why Technique

\subsection{W and 1H Technique}

This is the technique used in the failure situations. Most important steps are What: Whether all information was captured about the non compliance?

Who: Operators involved in activity are having training and experience?

When: Time of the non compliance happen?

Where: Whether the location is suitable for process activities?

Why: Reason for non compliance to be questioned?

After completing the $5 \mathrm{~W}$, conclusion will be questioned by How?

\subsection{Other Investigational Tools}

HER (Human Error Reduction) tool and Design of experiment tool are also useful to identify the root cause. These tools should be used as a supporting tool for investigation, but not as a major investigational tool.

\section{Impact on Product Quality, Final Classification and CAPA}

User department along with manufacturing sciences and R\&D will assess the impact on product quality. The impact assessment, final classification of the deviation will be done by QA. If impact identified on quality, necessary CAPA will be in place to avoid the 
non compliance or recurrence of the same or different type. CAPA is the tracking document or bridging document. CAPA initiation is the responsibility of user department which initiates deviation and effectiveness of the CAPA to be monitored by user department with the support of cross functional teams and QA. In case of any delay in investigation, an extension to the target date should be taken by user department from QA by using delay justification form (Annexure -4). All delay justification to be initiated by on or before the target date for closure. Failing to take delay justification on or before the target date will trigger a separate deviation and impact assessment for the same to be mentioned.

\section{Deviation Close Out}

Deviation to be closed by Head of the department of QA after the investigation and CAPA proposal, by mentioning the deviation details, root cause, impact on product quality and CAPA proposed if any along with the details like below.

1. Is it recurred deviation?: Yes/ No/ NA (Not Applicable)

2. Type of the deviation (Planned or Unplanned)

3. Category of the deviation (Critical/ Major/ Minor)

4. CAPA Proposed: Yes/ No/ NA

5. If CAPA proposed, CAPA number:

6. Supporting documents, if any:

Closed by Name, Sign \& Date:

\section{Annexure-1}

(Deviation management request form)

Annexure-1 Issued by: QA

Name of the person:

Date of issuance:

Type of deviation: Process/ Equipment

Note: If more than 2 forms are required please provide justification in remarks

Remarks:

Form requested by (Name, Sign \& Date):

User department head/ Section In-charge (Name, Sign \& Date):

Request received by QA (Name, Sign \& Date):

Deviation form issued by QA (Name, Sign \& Date):

Confirmation by the end user after receiving the form (Name, Sign \& Date):

Note: After receiving the deviation management form by user department, Annexure-1 should be archived with QA for trending the deviation management once in 03/06 months

\section{Annexure-2}

\section{Deviation form}

Department:

Date of occurrence:

Date of identification:

Date of initiation:

Deviation from: Process /Equipment/ Instrument/ Product/ Utilities/ others

Instrument number/ batch number/ equipment number/ others:

Deviation details in brief:

Deviation identified by (Name):

Deviation reported by (Name, Sign \& Date): 
Additional action or immediate additional action taken:

Review by user department head/in-charge:

Similar type of deviations observed previously: Yes/ No/ NA

If yes provide the details:

Deviation log in number:

Name of the person who assigned the number:

Sign \& Date:

Target date:

Initial classification: Critical/ Major/ Minor

Departments to be marked by QA:

RS/ NRA/ Production/ Manufacturing sciences/ QC/ QA/ Engineering/ Instrumentation/ others:

Number of departments by QA:

Name of the QA person:

Sign \& Date:

Review comments by NRS:

Review comments by IRS:

Review comments by Production:

Review comments by Manufacturing sciences:

Review comments by QC:

Review comments by QA:

Review comments by Engineering:

Review comments by Instrumentation:

Review comments by others:

Cumulative review by QA:

Investigation, impact assessment and Corrective action and preventive action by user:

User department Name, Sign \& Date:

Final classification: Critical/ Major/ Minor

CAPA reference number:

Review comments on Investigation, impact assessment and Corrective action and preventive action by NRS:

Review comments on Investigation, impact assessment and Corrective action and preventive action by IRS:

Review comments on Investigation, impact assessment and Corrective action and preventive action by Production:

Review comments on Investigation, impact assessment and Corrective action and preventive action by Manufacturing sciences:

Review comments on Investigation, impact assessment and Corrective action and preventive action by QC:

Review comments on Investigation, impact assessment and Corrective action and preventive action by QA:

Review comments on Investigation, impact assessment and Corrective action and preventive action by Engineering:

Review comments on Investigation, impact assessment and Corrective action and preventive action by Instrumentation:

Review comments on Investigation, impact assessment and Corrective action and preventive action by others:

Impact identified on product: Yes/ No/ NA

If yes, Please provide the level of impact: High/ Moderate/ Low/ NA

Correction action proposed to avoid the non compliance: Yes/ No/ NA

If yes, Please provide the details:

Preventive action proposed to avoid the recurrence: Yes/ No/ NA

If yes, Please provide the details: 
CAPA initiate on:

CAPA number:

CAPA number issued by Name, Sign \& Date:

Deviation close out:

List of supporting documents:

\section{Annexure - 3}

Deviation management register

S. No:

Date of deviation occurred:

Initiated by department:

Deviation number:

Batch/ Equipment/ Instrument/ Document/ other codes:

Deviation details:

Log in by QA (Name, Sign \& Date):

Classification (Critical/Major/Minor)

Impact on product (Yes/No/ NA)

Date of closure:

Closed by Name, Sign\& Date:

Verified by Name, Sign \& Date:

Remarks:

\section{Annexure - 4}

Delay justification form

Quality element: Deviation/ Change control/ OOS (Out Of Specification)/ OOT (Out Of

Trend)/ others

Specify if others:

Deviation/ Change control/ OOS/ OOT/ others number:

Reason/ Justification for delay in closure:

Whether delay has any impact on product quality (Yes/ No/ NA):

Delay: First delay/ Second delay/ Third delay

If first delay, mention the initial committed target date:

If second delay, mention the first target date:

If third delay, mention the second target date:

Next target date:

Name of initiator, Sign\& Date:

Reviewed by user department head (Name, Signe \& Date):

Approved by QA (Name, Sign \& Date):

\section{Conclusion}

All non conformances during manufacturing process are logged by deviation. Immediate corrective action is proposed to nullify the impact on product quality. All deviations are investigated for the proper root cause. Appropriate CAPA is proposed to avoid the recurrences. Best industrial quality system practice will have all information furnished above. Deviations will be initiated for system improvements and where impact assessment on system and product quality is required. An efficient quality management system does not require deviations for typo errors where supporting documents are available and which are not impacting process or product quality. In those cases, a clear justification to be provided by user department head/ in-charge before closure of batch 
processing record/ batch manufacturing record/ checklist/ Annexure of standard operating procedure and duly approved by QA personnel.

\section{Acknowledgements}

PVD Sowjanya Kumari cordially conveying their regards to Dr. Peravali Jawahar Babu and Mr. Tanniru Bala Sundara Rao for the information provided.

\section{References}

[1] T. Daya, D. Surendra Naidu, N. Lavanya Reddy, K. Seetha Ram and R. Ganapathy, "Current Prospects of Change Management in Biopharmaceutical Quality Systems - A Holistic Cumulative Review", International Journal of Bio-Science and Bio-Technology, vol. 9, no. 5, (2017), pp. 1-12.

[2] https://www.fda.gov/.

[3] http://www.ema.europa.eu/ema/.

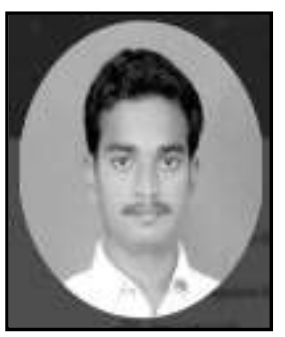

Ganesh Tsavatapalli, a post graduate biotechnologist with $10+$ years of industrial experience in various fields including production and manufacturing sciences. Currently working in an international organization which contributes for human health. His scientific helping nature in the way of ideas and ideology and; outstanding knowledge towards industrial sciences are more worthy. He is also one of the role model for upcoming industrial biotechnologists.

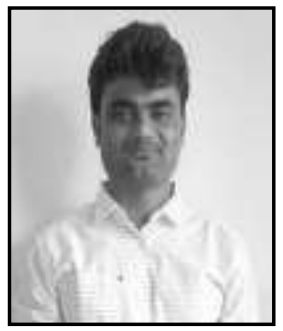

Sudheer Reddy Billipelli, a post graduate microbiologist with 06 years of industrial experience in the areas of viral vaccines, bacterial vaccines and biosimilars. He is currently working in a premier biotech company in India.

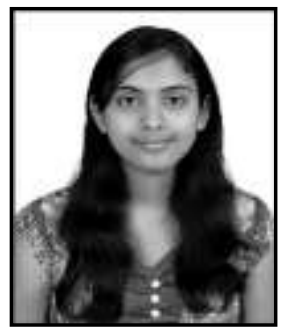

Lavanya Reddy $\mathbf{N}$, a young aspirant with an engineering degree in biotechnology pursuing Post graduate diploma in Pharmaceutical Regulatory affairs (PGRA). Currently working on quality systems of a renowned biopharmaceutical industry.

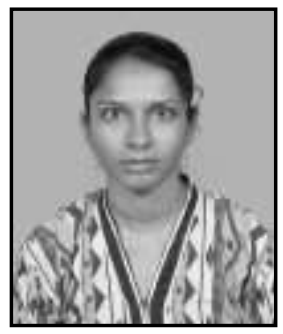

Sandhya Prathapaneni, completed her M.Sc chemistry from reputed organization. She has versatile knowledge in academics and on quality compliance. 


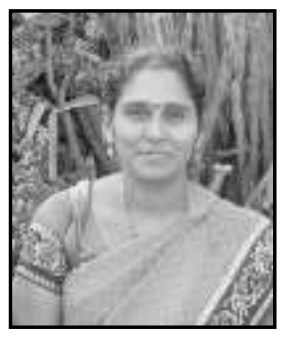

Rajeswari Ramayanam, a qualified post graduate physical science teacher having versatile knowledge on industrial current good manufacturing practices.

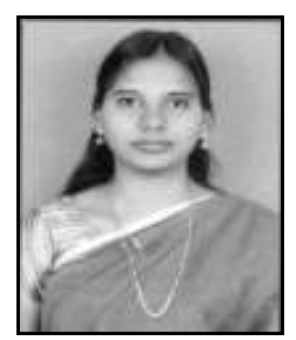

Dr. PVD Sowjanya Kumari, completed her Masters in Microbiology from Andhra University. Currently working as Head of the department, Microbiology at MVRPG college, Vishakapatnam. She has $15+$ years of experience and has publications in different national and international journals with good impact factor and indexation along with Dr. Seetha Ram Kotra. 